\title{
Efeitos da terapia de reposição hormonal com raloxifeno e risedronato na reparação óssea de ratas com osteopenia"
}

\section{"Effects of hormone replacement therapy with raloxifene and risedronate in bone repair of female rats with osteopenia"}

\author{
Aretha Graziela Vilela de SOUSA \\ Bolsista de Iniciação Científica (FAPESP - 05/51658-0) - Departamento de Biociências e Diagnóstico Bucal - Faculdade \\ de Odontologia de São José dos Campos - Universidade Estadual Paulista - UNESP - São José dos Campos - SP - Brasil
}

\section{Levy Anderson César ALVES}

Aluno de Iniciação Científica - Departamento de Biociências e Diagnóstico Bucal. - Faculdade de Odontologia de São José dos Campos - Universidade Estadual Paulista - UNESP - São José dos Campos - SP - Brasil.

\section{Rosilene Fernandes da ROCHA}

Professora Adjunta - Disciplina de Farmacologia - Departamento de Biociências e Diagnóstico Bucal - Faculdade de Odontologia de São José dos Campos - Universidade Estadual Paulista - UNESP - São José dos Campos - SP - Brasil

\section{Mari Eli Leonelli de MORAES}

Professora Doutora - Disciplina de Radiologia - Departamento de Diagnóstico e Cirurgia - Faculdade de Odontologia de São José dos Campos - Universidade Estadual Paulista - UNESP - São José dos Campos - SP - Brasil

\section{Valéria Abrantes Pinheiro CARVALHO}

Professora Doutora - Disciplina de Farmacologia - Departamento de Biociências e Diagnóstico Bucal - Faculdade de Odontologia de São José dos Campos - Universidade Estadual Paulista - UNESP - São José dos Campos - SP - Brasil

\begin{abstract}
Resumo
O objetivo do estudo foi de analisar radiograficamente os efeitos do risedronato e do cloridrato de raloxifeno na reparação óssea de ratas osteopênicas. Utilizou-se cinqüenta ratas divididas em cinco grupos de 10 animais cada: (I) SHAM, (II) ovariectomizadas (OVZ), (III) OVZ e tratadas com risedronato $1 \mathrm{mg} / \mathrm{kg} / \mathrm{dia}$, (IV) OVZ e tratadas com raloxifeno $1 \mathrm{mg} /$ $\mathrm{kg} /$ dia e (V) OVZ e tratadas com raloxifeno $3 \mathrm{mg} / \mathrm{kg} / \mathrm{dia}$. Após trinta dias da ovariectomia executaram-se lesões ósseas de $3 \mathrm{~mm}$ de $\varnothing$ nas tíbias direitas. Após 7 e 28 dias de tratamento as ratas foram sacrificadas e as tíbias avaliadas quanto à densidade radiográfica da região de reparação do defeito ósseo por meio do sistema digital RVG Trophy e programa Image Tool 2.03. Os dados da análise da densidade óptica radiográfica foram submetidos à ANOVA e Teste de Tukey $(\mathrm{p} \leq 0,05)$. Os resultados mostraram que aos 28 dias os grupos I e V apresentaram valores de densidade mineral óssea superiores e estatisticamente significantes em relação aos grupos II e IV e semelhantes ao grupo III. Além disso, o grupo III obteve densidade mineral óssea estatísticamente superior ao grupo II e semelhante ao grupo IV. Concluiu-se que o raloxifeno na dose de $3 \mathrm{mg} / \mathrm{kg} / \mathrm{dia}$ e o risedronato na dose de $1 \mathrm{mg} / \mathrm{kg} / \mathrm{dia}$, aceleraram o processo de reparação óssea, apresentando efeito semelhante ao grupo SHAM. O efeito do raloxifeno na reparação óssea mostrou-se dose-dependente.
\end{abstract}

\section{UNITERMOS}

Risedronato; raloxifeno; regeneração óssea; osteoporose; osteopenia; terapia de reposição hormonal; densidade óptica radiográfica. 


\section{INTRODUÇÃO}

Atualmente, a expectativa de vida da população brasileira tende a aumentar, sobretudo nas próximas décadas. O crescente número de indivíduos na faixa de idade acima dos 65 anos, ao contrário do que ocorre com outros grupos etários, tem gerado, na conduta dos profissionais da área da saúde, uma grande preocupação com a qualidade de vida dos idosos e, consequientemente, com a prevenção de patologias presentes e características do processo de envelhecimento.

De acordo com Castro \& Uyeno $^{6}(2000)$ e Piai ${ }^{19}$ (2005) as alterações decorrentes da senescência, a perda de massa muscular, o enfraquecimento do sistema imunológico, o declínio da acuidade visual são marcantes, bem como doenças crônicas como hipertensão, artrite, diabetes e problemas vasculares.

Assim também, distúrbios como a osteoporose, uma patologia sistêmica e progressiva, caracterizada pelo desequilíbrio entre atividade osteoblástica e osteoclástica, sendo esta última predominante neste processo, podem, eventualmente, levar à perda de massa considerável e deterioração da microarquitetura óssea. Nesta condição, a remodelação e a atividade metabólica óssea são alteradas (COSMAN $\left.{ }^{8}, 2005\right)$.

As projeções divulgadas, no que diz respeito à prevalência populacional da osteoporose para os próximos anos, como referido por $\mathrm{Amadei}^{4}$ (2006), estimam que 200 milhões de pessoas em todo o mundo serão afetadas por esta doença, referida como a mais importante doença músculo-esquelética e não-artrítica. No Brasil, 70 mil indivíduos apresentam, anualmente, fratura do colo do fêmur, sendo que pelo menos $20 \%$ morrem de complicações nos primeiros seis meses e metade perdem a independência em suas atividade diárias. Além disso, aproximadamente $30 \%$ destes pacientes necessitarão de cuidados institucionais ou de enfermagem domiciliar.

Sendo assim, a diminuição dos níveis de estrogênio constitui fator determinante e responsável pela gênese da osteopenia e osteoporose após a menopausa (COMSPSTON $^{7}, 2001$, ANBINDER $^{2}$ et al; 2006 e $\mathrm{AMADEI}^{4}$ et al; 2006). Os osteoclastos, além de sua função osteolítica, desempenham importante papel no desenvolvimento e crescimento ósseos ao liberar esses fatores de matriz extracelular mineralizada. Em fase de reabsorção, os osteoclastos ativados degradam tanto os componentes minerais quanto os orgânicos, pela secreção de enzimas ácidas e hidrolíticas, liberando fragmentos minerais ósseos e de colágeno. Os osteoclastos são estimulados por calcitriol, paratormônio (PTH), TNF, prostaglandinas $\mathrm{E}_{2}$, além das IL-1, 11 e 6 , e são inibidos por IL-4 e 13. (IWANIEC ${ }^{10}$ et al; 2001 e IWANIEC ${ }^{11}$ et al; 2002). Amadei ${ }^{4}$ e colaboradores (2006) descreveram a fisiopatologia da osteoporose como um evento complexo, envolvendo liberação de fatores de crescimento e citocinas. Mundy ${ }^{15}$ (1995) sugeriu que a interleucina 6 (IL-6) seria a citocina de maior importância na perda óssea quando ocorrem disfunções gonodais, entretanto seu papel parece ainda incerto, embora a IL-1, o fator de necrose tumoral (TNF) e Il-11 também possam participar dessa perda causada pela deficiência estrogênica.

Por essa razão, as terapias farmacológicas de reposição hormonal ou com substâncias estabilizadoras do processo reabsortivo, representam importantes estratégias no campo da saúde pública, pois têm, também, por objetivo impedir a perda adicional de osso e diminuir a probabilidade de futuras fraturas, além de prevenir o aparecimento de distúrbios cardiovasculares e, até mesmo, psíquicos decorrentes da deficiência hormonal na pós-menopausa.

Os bisfosfonatos, (Figura 1), são freqüentemente utilizados no tratamento de várias doenças ósseas. Entre eles temos o risedronato sódico considerado um dos mais potentes entre os disponíveis no mercado (SZEJNFELD $\left.{ }^{26}, 2000\right)$. Ao se fixarem na área da borda em escova dos osteoclastos, inibem a ação de desmineralização óssea enzimática (OLIVEIRA ${ }^{17}$, 2003). O risedronato liga-se à hidroxiapatita do tecido ósseo e inibe a sua reabsorção. A renovação óssea é

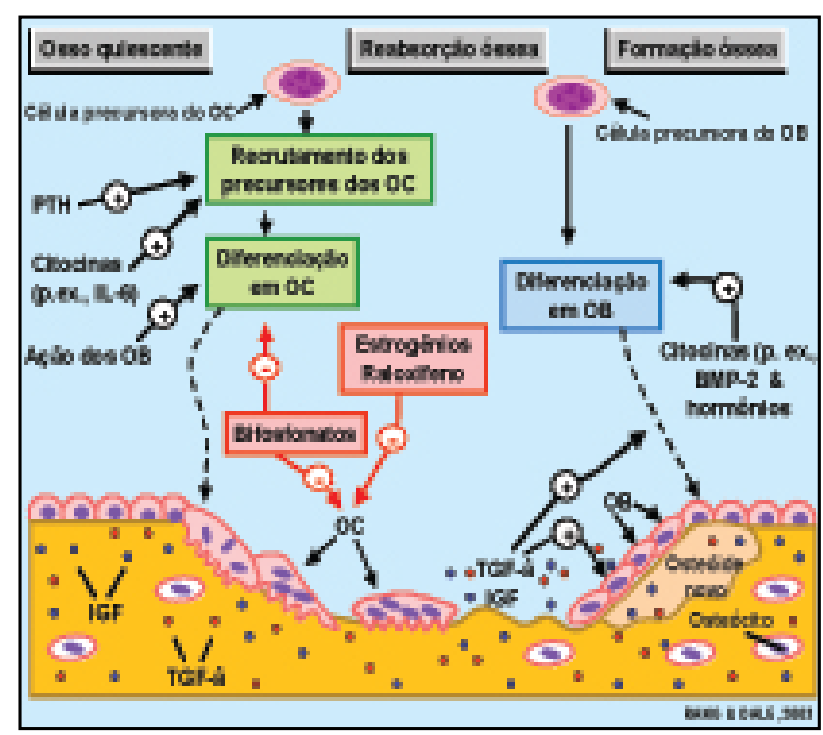

FIGURA 1 - Mecanismo de ação do raloxifeno e dos bisfosfonatos (risedronato). 
reduzida, enquanto a atividade osteoblástica e a mineralização são preservadas $\left(\mathrm{OTOMO}^{18}\right.$ et al; 2004; PIAI $^{19}$ et al; 2005 e ANBINDER ${ }^{2}$ et al; 2006). Estudos com risedronato demonstraram potente atividade anti-osteoclástica, com aumento da massa óssea de modo dose-dependente e alguns autores sugerem que os bisfosfonatos induzem a apoptose de osteoclastos (IWATA $^{12}$ et al; 2006).

Por outro lado, refere-se na literatura atual, o uso de "SERMs", moduladores seletivos dos receptores de estrogênios, utilizados na terapêutica do câncer de mama como o tamoxifeno, e entre estes o raloxifeno (subgrupo do tamoxifeno), utilizado para prevenção e tratamento da osteoporose (REGINSTER ${ }^{21}$ et al; 2004; SLIWINSKI $^{23}$ et al; 2005 e ROGERS ${ }^{22}$ et al; 2007). O referido fármaco é classificado como "SERM" de segunda geração, apresentando efeito tecido-específico nos órgãos-alvo de ação do estrógeno (ARMAMENTO-VILLARREAL ${ }^{5}$ et al; 2005; HERTRAMPF ${ }^{9}$ et al; 2006 e NARAYANA ${ }^{16}$ et al; 2006). Sua seletividade tecidual ocorre devido a diversos mecanismos de interação com diferentes receptores de estrógenos, além da distribuição diferencial destes receptores nos diversos tecidos (SLIWINSKI ${ }^{23}$ et al; 2005; $\mathrm{KOMM}^{13}$ et al; 2005 e SAMJEN ${ }^{24}$ et al; 2006).

Os mecanismos pelos quais os "SERMs" têm efeito antagonista/agonista de forma seletiva dos tecidos ainda não são completamente conhecidos (Figura 1). Alguns estudos sugerem que o raloxifeno é capaz de estimular as vias estrogênicas por meio do receptor específico. Os receptores de estrógeno pertencem a uma grande família de receptores nucleares, ER $\alpha$ e $\mathrm{ER} \beta$, sendo que o receptor alfa predomina nos órgãos reprodutores (mama e útero), enquanto que o receptor beta predomina no osso e sistema cardiovascular. $\mathrm{O}$ raloxifeno parece interagir com receptores beta, ativando múltiplas funções transcricionais, mas não é capaz de ativá-las via receptor alfa, podendo ser este um dos mecanismos pelos quais este fármaco exerce ação diferencial nos tecidos (KOMM ${ }^{13}$ et al; $2005 \mathrm{e}$ COSMAN $^{8}$ et al; 2005).

Diante dos efeitos acima descritos, o raloxifeno representa, dentre os "SERMs", ótimas perspectivas para sua utilização na terapia de reposição hormonal, considerando seus efeitos benéficos sobre o metabolismo ósseo e manutenção da massa óssea já relatados (IWANIEC $^{11}$ et al; 2001e ARMAMENTO-VILLARREAL ${ }^{5}$ et al; 2005), mas, sobretudo, por sua indicação de uso clínico em pacientes que apresentam histórico familiar de neoplasias mamárias e/ou endometriais, fatores que contra-indicam a reposição hormonal nos moldes clás- sicos, como até hoje aplicados. Desta forma o objetivo do presente estudo foi de analisar comparativamente os efeitos do raloxifeno em dois diferentes esquemas posológicos e do risedronato na dose de $1 \mathrm{mg} / \mathrm{kg} / \mathrm{dia}$ sobre o reparo ósseo de defeitos cirúrgicos em tíbias de ratas Wistar por meio da análise da densidade óptica radiográfica do osso neoformado.

\section{Material e mÉtodos}

Para o presente trabalho, foram utilizadas cinqüenta ratas Wistar com noventa dias de idade e peso aproximado de $250 \mathrm{~g}$ manipuladas de acordo com os Princípios Éticos para a experimentação Animal (CEP: Proc 009/2004-PA da Faculdade de Odontologia do campus de São José dos Campos). Os animais foram mantidos em gaiolas em temperatura ambiente, recebendo ração e água ad libitum. Estes foram divididos em cinco grupos de dez animais: (I) ratas (SHAM); (II) ratas ovariectomizadas (OVZ); (III) ratas OVZ e tratadas com Risedronato $1 \mathrm{mg} / \mathrm{kg} / \mathrm{dia}$; (IV) ratas OVZ e tratadas com Raloxifeno $1 \mathrm{mg} / \mathrm{kg} / \mathrm{dia}$ e (V) ratas OVZ e tratadas com Raloxifeno $3 \mathrm{mg} / \mathrm{kg} / \mathrm{dia}$.

As ratas dos grupos II, III, IV e V foram ovariectomizadas (Figura 2A) e após trinta dias do procedimento, foram confeccionadas lesões de $3 \mathrm{~mm}$ de diâmetro até o limite da medula óssea no terço proximal da tíbia com auxilio de broca esférica $\mathrm{n}^{\circ} 6 \mathrm{em}$ motor de baixa rotação, sob irrigação constante com soro fisiológico ( $\mathrm{NaCl}$ 0,9\%) (Figura 2B). Em seguida, ao grupo III foi administrado $1 \mathrm{mg} / \mathrm{kg} / \mathrm{dia}$ de risedronato, ao grupo IV $1 \mathrm{mg} / \mathrm{kg} / \mathrm{dia}$ de raloxifeno e ao grupo V $3 \mathrm{mg} / \mathrm{kg} /$ dia de raloxifeno (Figura 2C). Após 7 e 28 dias de tratamento, os animais foram sacrificados em número de 5, por período experimental.

\section{MÉtodo Radiográfico}

Para as incidências radiográficas das tíbias foi utilizado o aparelho de raios X digital 765 DC@ Gendex com 65 kVp, 7mA, e 0,032s de tempo de exposição. Para captação das imagens, utilizou-se um sistema de radiografia digital direto, que emprega o dispositivo de carga acoplada (CCD): o RVG (Trophy Radiologie, Vincennes, Toulose, France). Este sistema digital foi acoplado a um computador Pentium III com 1,4 GHz, $128 \mathrm{Mb}$ de memória, HD 40Gb, Monitor LG de 17". $\mathrm{O}$ sensor $\mathrm{CCD}$ foi fixado à uma mesa com o cilindro do aparelho de raios $\mathrm{X}$ e posicionado a uma distância focal de $40 \mathrm{~cm}$, de forma que o feixe central de raios $\mathrm{X}$ incidisse perpendicularmente ao sensor. Cada peça 

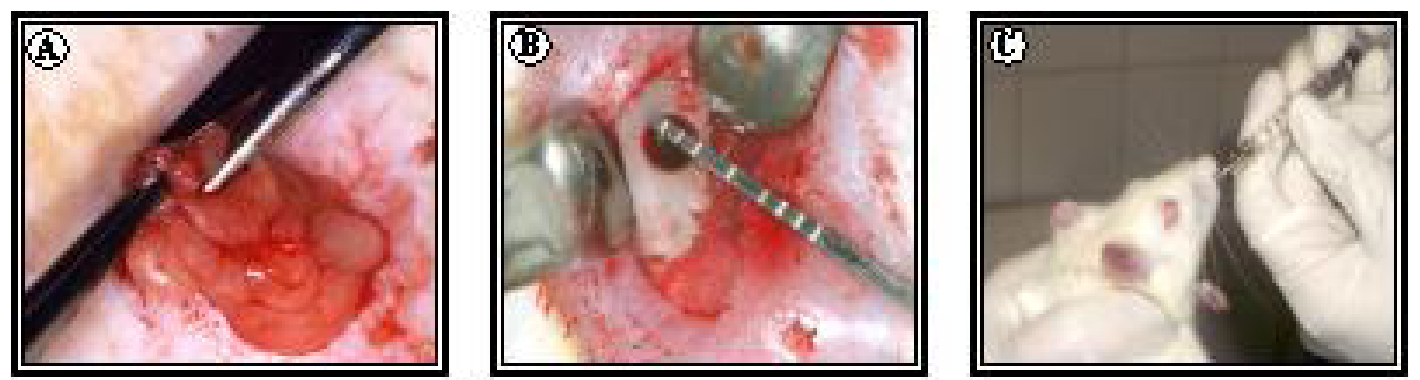

FIGURA 2 - A - Procedimento de ovariectomia. B- Defeito ósseo cirúrgico. C - Gavagem

anatômica foi colocada no sensor com o defeito ósseo ocupando a porção central deste. Na porção lateral do sensor foi colocado uma escala de alumínio, em que cada degrau tinha $0,5 \mathrm{~mm}$ de espessura, para a comparação do grau de densidade e as imagens obtidas foram armazenadas no formato TIFF.

Posteriormente, a análise da densidade óptica foi realizada no programa Image Tool $2.03^{\circledR}$, utilizando-se a ferramenta histograma (densidade óptica x número de pixels), em que demarcou-se a região central do defeito ósseo. Desta maneira, obteve-se um gráfico bidimensional, fornecendo os valores de cinza da imagem radiográfica. Foram realizadas duas leituras das médias das densidades de cada imagem radiográfica, no intervalo de uma semana cada, pelo mesmo examinador. Os valores obtidos foram submetidos à análise estatística.

\section{Análise estatística}

Os dados obtidos proporcionaram a análise comparativa das leituras ópticas dos grupos, utilizando-se métodos estatísticos descritivos por meio de tabelas, gráficos e parâmetros de média e desvio padrão. Para inferência populacional na análise dos grupos, na determinada incidência de $0.032 \mathrm{~s}$, para densidade óptica utilizou-se Análise de Variância (ANOVA). Quando esta indicou diferenças estatisticamente significantes, foi utilizado o teste de Tukey (5\%) para comparações múltiplas, ambos, com auxílio do programa STATISTIX 7.0 (2000 - Analitical Software Co.). Em todos os casos adotou-se nível de significância de 5\%.

\section{Resultados}

As imagens radiográficas digitais obtidas das peças cirúrgicas (Figuras 3 e 4), foram analisadas e descritas de acordo com suas variações de densidade óptica radiográfica. No período de setes dias de obser- vação (Figura 3) notou-se, nos grupos experimentais, densidade radiográfica relativamente uniforme na extensão da loja cirúrgica com limites bem definidos e ressaltou-se, na área central, maior radiotransparência em relação à área periférica. No período de vinte e oito dias de tratamento (Figura 4) a análise da densidade mineral óssea revelou limites pouco precisos com radiopacidade uniforme na região central e periférica, permitindo constatar a formação de tecido de reparação, sobretudo nos espécimes dos grupos I, III, IV e V (Figuras 4 I, 4 III, 4 IV e 4 V). O grupo II (Figura 4 II) apresentou limites precisos da loja cirúrgica, bem como baixo grau de densidade óptica radiográfica, caracterizando reparação óssea incompleta na região, sobretudo no que se refere à porção central do defeito, onde notou-se significativa radiolucidez.

Os resultados desta pesquisa mostraram que os animais dos grupos I, III, IV e V apresentaram um aumento estatisticamente significante na densidade óssea mineral no período compreendido entre sete e vinte e oito dias de observação, de acordo com a representação esquemática em letras maiúsculas na tabela 1. O grupo II, por outro lado, não mostrou diferença estatisticamente significante entre os valores de densidade mineral óssea medidas aos sete e vinte e oito dias de observação.

Ainda de acordo com a tabela 1 , considerando a representação em letras minúsculas, a análise de reparação aos setes dias de observação revelou valores de densidade mineral semelhantes entre todos os grupos experimentais. Em contrapartida, aos vinte e oito dias, pôde-se constatar que as médias da densidade óssea mineral foram semelhantes entre os grupos I, III e V, assim como entre os grupos III e IV.

Conforme o gráfico da figura 5 e levando-se em consideração os dados obtidos, pôde-se destacar um desempenho superior dos grupos III e V no que se refere ao processo de reparação óssea no período de vinte e oito dias das terapias medicamentosas propostas. 

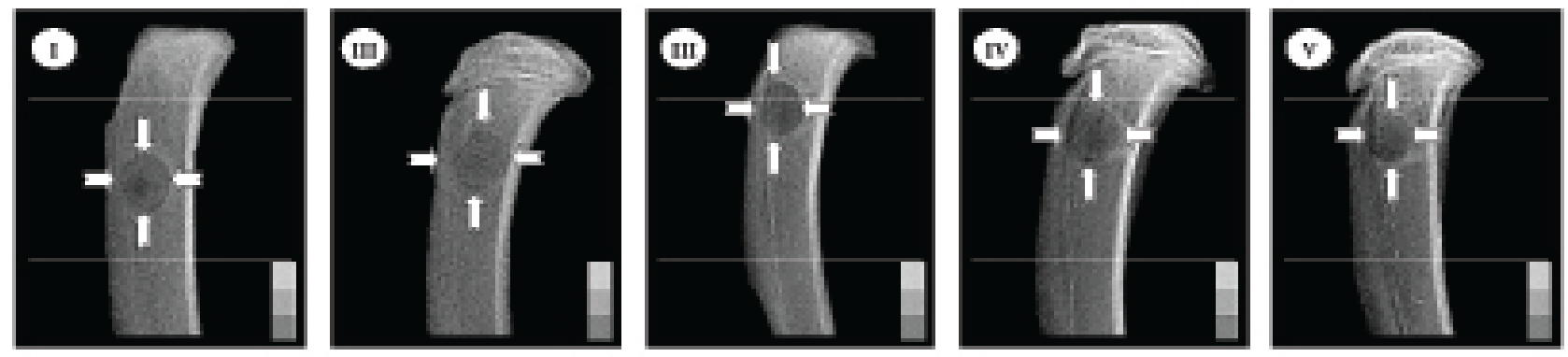

FIGURA 3 - Radiografias das tíbias das ratas dos grupos I, II, III, IV e V mostrando a reparação óssea aos sete dias. (setas). Escala de alumínio posicionada no canto inferior direito.
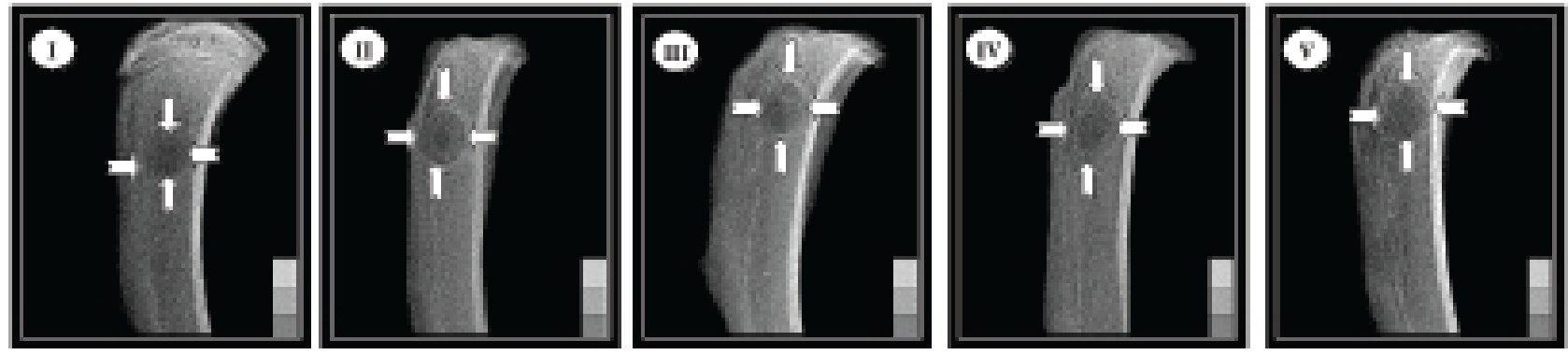

FIGURA 4 - Radiografias das tíbias das ratas dos grupos I, II, III, IV e V mostrando a reparação óssea aos vinte e oito dias dias. (setas). Escala de alumínio posicionada no canto inferior direito.

Tabela 1 - Médias ( $\bar{x}$ ) e desvio padrão $(\sigma)$ da densidade mineral óssea

\begin{tabular}{|c|c|c|c|c|c|c|c|c|c|c|}
\hline Grupos & I & & & & I & & I & & $v$ & \\
\hline Períodos & $\bar{x}$ & $\sigma$ & $\bar{x}$ & $\sigma$ & $\bar{x}$ & $\sigma$ & $\bar{x}$ & $\sigma$ & $\bar{x}$ & $\sigma$ \\
\hline 7 dias & $83,0 \mathrm{Aa}$ & $\pm 6,6$ & $\begin{array}{l}75,0 \\
\mathrm{Aa}\end{array}$ & $\pm 6,8$ & $\begin{array}{l}73,0 \\
\mathrm{Aa}\end{array}$ & $\pm 8,1$ & $\begin{array}{l}73,0 \\
\mathrm{Aa}\end{array}$ & $\pm 8,8$ & $\begin{array}{l}80,4 \\
\mathrm{Aa}\end{array}$ & $\pm 11,3$ \\
\hline 28 dias & $96,5 \mathrm{Bb}$ & $\pm 8,7$ & $\begin{array}{l}78,0 \\
\mathrm{Aa}\end{array}$ & $\pm 6,6$ & $\begin{array}{l}92,2 \\
\mathrm{Bbc}\end{array}$ & $\pm 7,8$ & $\begin{array}{l}92,2 \\
\text { Aac }\end{array}$ & $\pm 5,1$ & $\begin{array}{l}95,3 \\
\mathrm{Bb}\end{array}$ & $\pm 8,6$ \\
\hline
\end{tabular}

${ }^{*}$ Medidas em pixels

** Médias seguidas de letras distintas (maiúsculas na vertical e minúsculas na horizontal) diferem entre si. (Vieira $\left.{ }^{27}, 2004\right)$.

\section{Dıscussão}

O termo osteopenia refere-se à densidade mineral óssea diminuída, sendo um sinal clínico que pode estar associado à osteoporose primária ou à osteoporose secundária. As modalidades de osteoporose prevalentes na população são aquelas denominadas osteoporose tipo I ou pós-menopáusica e osteoporose tipo II ou senil. Dessa maneira, a osteoporose pode ser definida pelo elevado risco de fratura por fragilidade óssea aumentada pela redução na quantidade de tecido ósseo por unidade de volume (SZEJNFELD ${ }^{26}, 2000$ ).

Dentro deste contexto, a osteoporose do tipo I é característica de mulheres com queda dos níveis estrogênicos no período pós-menopáusico. O tratamento ideal da osteoporose é ainda o preventivo e baseia-se 


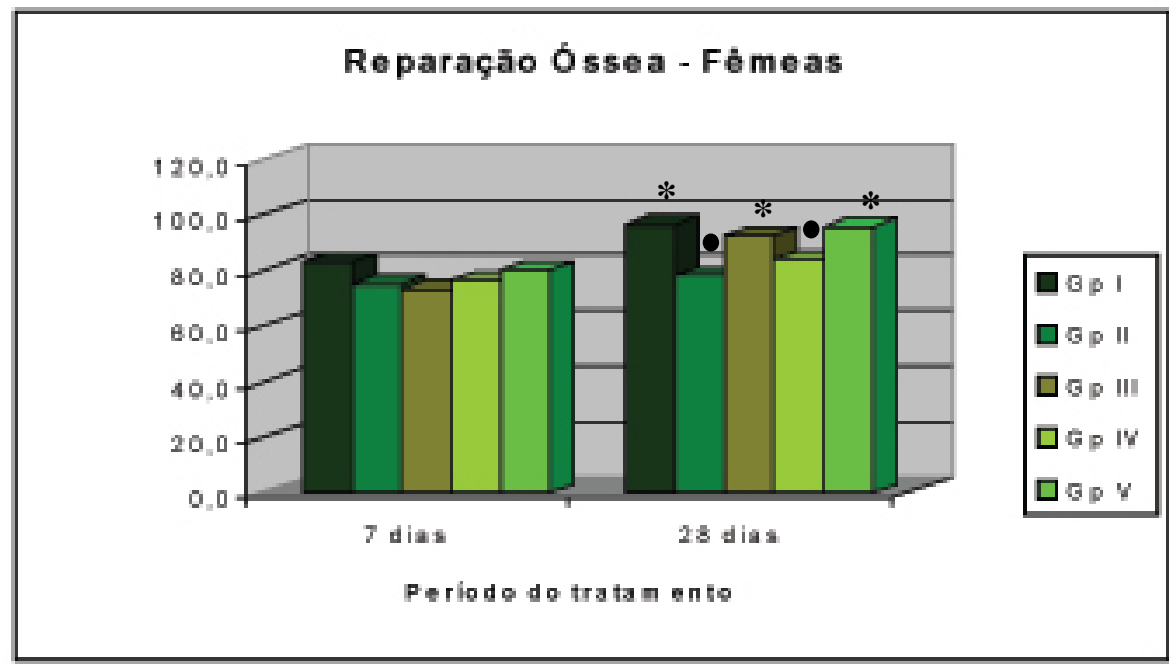

FIGURA 5 - Gráfico das médias de densidade óptica (pixels) nos períodos experimentais. Grupos estatisticamente semelhantes possuem o mesmo símbolo $(\bullet, *)$

na otimização do pico de massa óssea na juventude, na redução das perdas ao longo da vida e na prevenção de quedas (AMADEI ${ }^{4}$ et al; 2006). A reposição estrogênica no climatério é fundamental na prevenção da osteoporose e até o momento, os esquemas disponíveis para tratamento da mesma trazem resultados distantes do ideal para a cura completa da doença. Além disso, os indivíduos portadores de osteoporose ou osteopenia apresentam deficiências na reparação óssea, decorrente de fraturas ou defeitos cirúrgicos com o objetivo de colocação de implantes dentários, por exemplo (SZEJNFELD $\left.{ }^{26}, 2000\right)$.

A pesquisa realizada baseou-se em protocolos experimentais definidos em trabalhos que estudaram a reparação óssea em animais com osteopenia. Com relação ao local escolhido para realização do defeito ósseo, o osso adotado foi a tíbia, pois segundo Sou$\mathrm{za}^{25}$ e colaboradores (2005) não existe diferença na utilização deste osso para o mandibular diretamente relacionado à área odontológica, pois embora apresentem características embrionárias diferentes possuem processo de reparo e remodelação semelhantes (AMADEI $\left.{ }^{3}, 2004\right)$.

Entretanto, o maior problema está na determinação do tamanho crítico ideal, definido como o menor possível, sem quem este regenere-se espontaneamente durante o tempo de vida do animal ou pelo o período de observação (NARAYANA ${ }^{16}$ et al; 2006 e $\mathrm{SAMJEN}^{24}$ et al; 2006). Ainda não está bem determinado na literatura o tamanho dos defeitos realizados em ossos longos $\left(\right.$ SOUZA $\left.^{23}, 2005\right)$, por isso, optamos por um defeito de $3 \mathrm{~mm}$ de diâmetro, em função do tamanho dos animais, evitando também a fratura das tíbias (AMADEI $\left.{ }^{3}, 2004\right)$.

Assim também a idade ideal para que se realize a ovariectomia em ratas é de 3 meses de idade, pois nesse período, as mesmas encontram-se em fase reprodutiva (IWATA ${ }^{12}$ et al; 2006). Outro aspecto importante a ser levado em conta na análise do protocolo de indução de osteopenia por castração em animais experimentais, refere-se à determinação do período pós-cirurgia de castração necessário para o desenvolvimento do quadro de osteopenia. Assim sendo, inúmeros autores Amadei ${ }^{3}$ (2004), Amadei $^{4}$ et al. (2006), Iwaniec ${ }^{11}$ et al. (2002), Piai ${ }^{19}$ et al. (2005) referiram protocolos de períodos extremamente variados, por vezes divergentes, sendo, portanto, extremamente difícil escolha baseada em padrão da literatura do período pós-castração suficiente para indução de osteopenia. Existem na literatura trabalhos que indicam que três a quatro meses de castração (OTOMO ${ }^{18}$ et al; 2004 e REGINTER ${ }^{21}$ et al; 2004) são necessários para estabelecer-se um quadro de osteopenia em ratas com diminuição da DMO (densidade mineral óssea) e volume ósseo (ARMAMENTO-VILLAREAL ${ }^{5}$ et al; 2005; IWATA ${ }^{12}$ et al; 2006 e HERTRAMPF ${ }^{9}$ et al; 2006). No presente trabalho, o período de trinta dias pós-cirurgia de ovariectomia adotado proporcionou aumento quantitativo da densidade óptica radiográfica da região do defeito ósseo em reparação no grupo sham aos sete dias e estatisticamente maior no mesmo grupo aos vinte e oito dias. 
Quanto aos efeitos do risedronato e do raloxifeno no processo de reparação óssea de ratas, nossos resultados mostraram que os animais tratados com risedronato na dose de $1 \mathrm{mg} / \mathrm{kg} /$ dia tiveram um aumento estatisticamente significativo, entre sete e vinte e oito dias de tratamento, na densidade mineral óssea, ou seja, no valor médio de pixels. Estes dados confirmam os resultados de IWATA ${ }^{12}$ e colaboradores (2006) que estudaram os bisfosfonatos observando que são tão efetivos quanto estrógeno na inibição da reabsorção e turnover ósseo, evitando que este tecido, na ovariectomia induzida, apresente perda da arquitetura óssea.

Assim também, autores como Cosman ${ }^{8}$ (2005), Piai $^{19}$ et al. (2005) e Oliveira ${ }^{17}$ (2003), ao estudarem os efeitos do risedronato no metabolismo ósseo e/ou no processo de reparação de fraturas tanto de humanos quanto de animais experimentais, puderam comprovar no conjunto de seus resultados estatísticos e qualitativos um aumento na densidade mineral óssea, indicando efeito semelhante ao estrogênio por parte deste fármaco. Quanto aos dados obtidos referentes ao grupo tratado com raloxifeno 1 e $3 \mathrm{mg} / \mathrm{kg} / \mathrm{dia}$, observou-se, no presente trabalho, que as médias de densidade óptica radiográfica aos sete dias de reparação, assim como aos vinte e oito dias experimentais foram semelhantes ao grupo sham. Os dados obtidos nesta pesquisa confirmam aqueles encontrados por Reginster ${ }^{21}$ et al.(2004), Armamento-Villareal ${ }^{5}$ et al. (2005), Sliwinski ${ }^{23}$ et al. (2005), Narayana ${ }^{16}$ et al. (2006), Somjen ${ }^{24}$ et al. (2006), Rogers $^{22}$ et al. (2007), que constataram em seus trabalhos de pesquisa,utilizando raloxifeno em animais experimentais e humanos, o aumento e/ou preservação da DMO e diminuição do risco de fraturas. Nos estudos acima mencionados que mostraram clinicamente diminuição dos sintomas da osteoporose, as doses de raloxifeno obedeceram a protocolos que variaram de 60 a $120 \mathrm{mg}$ por períodos também variáveis entre 6 meses a 3 anos, em humanos.

Inúmeros pesquisadores como Oliveira ${ }^{17}$ (2003), IWATA $^{12}$ e colaboradores (2006), e Szejnfeld ${ }^{26}$ (2000), compararam os efeitos do risedronato com os do raloxifeno sobre o tecido ósseo e constataram que ambos diminuíram o risco de fratura, aumentaram a DMO, preveniram a perda óssea, porém, o risedronato foi inferior ao raloxifeno nos aspectos mencionados. Apenas nos estudos com animais tratados com risedronato associado ao PTH (IWANIEC ${ }^{10}$ et al; 2001 e IWANIEC ${ }^{11}$ et al; 2002), é que esta associação apresentou aumento na deposição mineral em relação ao raloxifeno isoladamente. No presente estudo, os defeitos ósseos dos animais tratados com raloxifeno demonstraram valores de radiopacidade semelhantes aos do grupo tratado com risedronato, tanto aos sete quanto aos vinte e oito dias de observação, de acordo, também, com os estudos acima mencionados.

Quanto aos efeitos do raloxifeno sobre a perda óssea em animais ovariectomizados testados por Hertrampf $^{9}$ et al. (2006) na dose de $3 \mathrm{mg} / \mathrm{kg} / \mathrm{dia}$ por sete semanas, quando comparado aos experimentos realizados em 2005 por Sliwinski ${ }^{23}$ e colaboradores testando raloxifeno na dose de $3 \mathrm{mg} / \mathrm{kg} /$ dia por oito semanas, observou-se que em ambos os trabalhos houve um ganho de DMO, em animais experimentais, pelo uso do raloxifeno. Da mesma forma em nossa pesquisa, a utilização de raloxifeno nas doses de 1 e $3 \mathrm{mg} / \mathrm{kg} / \mathrm{dia}$ apresentou, aumento estatisticamente significativo na densidade mineral óssea aos vinte oito dias de reparação, sendo que o raloxifeno na dose de $3 \mathrm{mg} / \mathrm{kg} / \mathrm{dia}$ obteve grau de radiopacidade semelhante ao do grupo sham no mesmo período experimental.

Quanto aos efeitos biológicos diferenciados em relação ao tempo para reparação do defeito ósseo, deve-se levar em conta as diferenças de comportamento farmacodinâmico entre os dois fármacos estudados. Assim sendo, sabe-se que o risedronato se liga à hidroxiapatita na superfície óssea, diminuindo o número e a atividade dos osteoclastos, inibindo a reabsorção óssea,sem provocar alterações na histologia óssea e permanendo neste tecido por longos períodos (SZEJNFELD $^{26}$, 2000). Foram demonstrados efeitos rápidos dos bisfosfonatos na permeabilidade dos osteoclastos ao cálcio, de modo semelhante ao que ocorre na zona de aderência dessas células. Diversos trabalhos constataram que bisfosfonatos adsorvidos à superfície óssea atuam diretamente sobre os osteoclastos, inibindo sua ação. Estes dados podem explicar os resultados de nossa pesquisa no que se refere, ao maior grau de densidade radiográfica apresentado pelo grupo III em relação ao grupo IV, aos vinte e oito dias de reparação óssea, apesar de não apresentar significância estatística. $\mathrm{O}$ dados do presente estudo demonstram a maior velocidade, ou seja, um menor período necessário ao início dos efeitos anti-reabsortivos do risedronato em relação ao raloxifeno, devido ao fato das moléculas de risedronato serem rapidamente fagocitadas pelos osteoclastos, provocando alterações morfofuncionais importantes nestas células

Quanto ao mecanismo de ação do raloxifeno, sabe-se que são moduladores seletivos do receptor de estrogênio (SERMs) apresentando ação antagonista sobre a mama e o útero e agonista sobre o metabolismo ósseo. Pesquisadores descreveram a presença de 
receptores de estrogênio em osteoblastos, sendo estes, portanto, considerados como células-alvo da resposta estrogênica. Embora os receptores de estrogênio estejam, predominantemente, nos osteoblastos, a principal atuação desse esteróide, reside na inibição da reabsorção. Parece que os esteróides sexuais e seus análogos promovem apoptose de osteoclastos. Desta maneira, estes inibiriam a liberação de fatores estimuladores dos osteoclastos ou aumentariam seus fatores inibitórios (MUNDY ${ }^{15}$ et al; 1995). A resposta biológica à ação estrogênica é um fenômeno saturável, pois o número de receptores por unidade de massa de tecido é limitado. O receptor de esteróide deve possuir alta afinidade, uma vez que a concentração sérica de hormônio se situa em torno de $10^{-10} \mathrm{M}$ a $10^{-8} \mathrm{M}$ (SZEJNFELD ${ }^{24}$ 2000). Além disso, estudos pregressos (REGINSTER ${ }^{21}$ et al; 2004; ARMAMENTO-VILLAREAL ${ }^{5}$ et al; 2005; NARAYANA ${ }^{16}$ et al; 2006 e $\mathrm{SOMJEN}^{24}$ et al; 2006)sugerem que os moduladores seletivos do receptor de estrogênio poderiam, ainda, agir em receptores específicos para eles, paralelamente à sua ligação com receptor de estrogênio (ROGERS ${ }^{22}$ et al; 2007).

Diante dessas afirmações, pode-se explicar que em nossos experimentos os grupos IV e $\mathrm{V}$ apresentaram uma média de densidade mineral óssea, aos vinte e oito dias, superior e estatisticamente significativa em relação aos 7 dias de reparação do mesmo grupo, mostrando que a necessidade de síntese de receptores específicos para atuação do raloxifeno, nos osteoblastos, faz com que haja necessidade de tempo maior que sete dias para observarmos o início dos efeitos metabólicos dos "SERMs" sobre o tecido ósseo.

Trabalhos de Allen ${ }^{1}$ et al. (2006) e Sliwinski ${ }^{23}$ et al. (2005) que utilizaram, risedronato 0,05 ou 0,10 $\mathrm{mg} / \mathrm{kg} / \mathrm{dia}$ e raloxifeno $0,50 \mathrm{mg} / \mathrm{kg} / \mathrm{dia}$ por doze meses em cães, mostraram que houve um aumento significativo de deposição mineral na superfície perióstica. Além disso, o raloxifeno levou uma melhora das propriedades mecânicas ósseas. Estes resultados podem explicar também os achados radiográficos em nossos estudos aos sete dias de observação, onde os grupos IV e V, tratados com raloxifeno 1 e $3 \mathrm{mg} / \mathrm{kg} /$ dia respectivamente, não diferiram estatisticamente dos demais grupos experimentais. Porém, de acordo com Iwaniec ${ }^{11}$ et al. (2002) e Cosman ${ }^{8}$ (2005) que relatam os efeitos positivos da terapia com raloxifeno na prevenção da perda óssea, nossos dados de densidade óptica radiográfica demonstraram, que aos 28 dias de observação o grupo $\mathrm{V}$ revelou grau de densidade mineral óssea maior e estatisticamente significante quando comparado aos grupos II e IV.

Assim, considerando que a TRH com estrogênio, aumenta o risco de desenvolver câncer; aliado ao fato de nossos resultados terem sidos considerados positivos e dose-dependentes, no que se refere aos efeitos do raloxifeno na reparação óssea de defeitos cirúrgicos, podemos apontar como sendo extremamente proveitosa a indicação do uso do raloxifeno como alternativa para a TRH em pacientes com risco alto para neoplasia de mama e útero, consistindo em achados importantes para a área odontológica, sendo valiosa alternativa terapêutica coadjuvante nos tratamentos que envolvem a necessidade de ganho ósseo.

\section{Conclusões}

- O raloxifeno $3 \mathrm{mg} / \mathrm{kg} / \mathrm{dia}$ e risedronato $1 \mathrm{mg} /$ $\mathrm{kg} / \mathrm{dia}$ foram eficazes na reparação óssea, após vinte e oito dias, apresentando graus de densidade mineral óssea estatisticamente maiores em relação ao grupo de ratas ovariectomizadas e semelhantes ao grupo SHAM.

- O raloxifeno, apresentou efeito dose-dependente, sendo a média de densidade mineral óssea maior e estatisticamente significante aos vinte e oito dias de observação, quando aplicado esquema posológico de $3 \mathrm{mg} / \mathrm{kg} / \mathrm{dia}$.

\section{Abstract}

The aim of this study was to analyse radiographically the effects of risedronate and raloxifene hydrochloride in bone repair of female rats with osteopenia. Fifty animals were divided in five groups of ten animals each: (I) SHAM, (II) Ovariectomised (OVZ), (III) OVZ and treated with $1 \mathrm{mg} / \mathrm{kg} /$ day risedronate, (IV) OVZ and treated with $1 \mathrm{mg} / \mathrm{kg} / \mathrm{day}$ raloxifene and (V) OVZ and treated with $3 \mathrm{mg} / \mathrm{kg} /$ day raloxifene. Thirty days after ovariectomy a $3 \mathrm{~mm}$ diameter bone deffect was made in the right tibia of the animals. After seven and twenty-eigth days of treatment, the animals were sacrificed in groups of five, and the tibias were analysed in terms of radiographic optical density by the digital system RVG Trophy and the Image Tool programme 2.03. All data collected were analysed by ANOVA and the Tukey's Test $(\mathrm{p}<=0,05)$. The results showed that after 28 days of treatment, groups I and V had greater and stastistically significant values of bone mineral density when compared to groups II and IV and similar values when compared to group III. 
Besides, the bone mineral density for group III was greater and stastistically significant when compared to group II and similar when compared to group IV. Hence, it could be concluded that $3 \mathrm{mg} / \mathrm{kg} / \mathrm{day}$ of raloxifene and $1 \mathrm{mg} / \mathrm{kg} / \mathrm{day}$ of risedronate, accelerated bone repair but showed similar effects to group I. In addition, it could be noticed that the effect of raloxifene in bone repair showed a dose-dependent relation.

\section{UNITERMS}

Risedronate; raloxifene; bone regeneration; osteoporosis; osteopenia; hormone replacement teraphy; radiographic optical density

\section{REFERÊNCIAS}

1. Allen MR, Follet H, Khurana M, Sato M, Burr DB. Antiremodeling agents influence osteoblast activity differently in modeling and remodeling sites of canine rib. Calcif Tissue Int. 2006; 78 (4): 255-61.

2. Anbinder AL, Prado MA, Spalding M, Balducci I Carvalho YR, Rocha RF. Estrogen deficiency and periodontal condition in rat- A radiographic and macroscopic study. Braz Dent J. 2006; 17 (3): 201-7.

3. Amadei SU. Estudo Comparativo dos efeitos da Isoflavona e da Ipriflavona na Reparação óssea em tíbias de ratas ovariectomizadas [dissertação]. São José dos Campos : Faculdade de Odontologia de São José dos Campos, Universidade Estadual Paulista; 2004.

4. Amadei SU, Silveira VA, Pereira AC, Carvalho YR, Rocha RF. A influência da deficiência estrogênica no processo de remodelação e reparação óssea. J Bras Patol Méd Lab. 2006 Fev; 1 (42) :7-15.

5. Armamento-Villareal R, Sheikh S, Nawaz A, Napoli N, Mueller C, Halstead LR, et al. A New Selective Estrogen Receptor Modulator, CHF 4227.01, Preserves Bone Mass and Microarchitecture in Ovariectomized Rats. J Bone Miner Res. 2005 Dec; 20: 2178 -88.

6. Castro ML, Uyeno MNO. Osteoporose no homem. In: Bandeira F.Osteoporose. Rio de Janeiro: Medsi; 2000; 147-165.

7. Compstom NJE. Sex steroids and bone.Physiol Rev. 2001, 81 (1): 41947.

8. Cosman F. The prevention and of osteoporosis: a review. Med Gen Med. 2005 May ; 7 (2): 73.

9. Hertrampf T, Degen GH, Kaid AA, Laudenbach-Leschowsky U, Seibel J, Di Virgilio AL, Diel P. Combined effects of physical activity, dietary isoflavones and 17beta-estradiol on movement drive, body weight and bone mineral density in ovariectomized female rats. Planta med. 2006 May; 72 (6): 484-87.

10. Iwaniec UT, Samnegard E, Cullen DM, Kimmel DB. Maintenance of cancellous bone in ovariectomized, human parathyroid hormone [hPTH(1-84)]-treated rats by estrogen, risedronate, or reduced $\mathrm{hPTH}$. Bone. 2001 Oct; 29 (4): 352-60.

11. Iwaniec UT, Mosekilde L, Mitova-Caneva NG, Thomsen JS, Wronski TJ. Sequential treatment with basic fibroblast growth factor and PTH is more efficacious than treatment with PTH alone for increasing vertebral bone mass and strength in osteopenic ovariectomized rats. Endocrinology. 2002 Jul; 143 (7): 2515-23.

12. Iwata K, Li J, Follet H, Phipps RJ, BurrDB. Bisphosphonates suppress periosteal osteoblast activity independently of resorption in rat femur and tibia. Bone. 2006 Nov; 39 (5): 1053-58.

13. Komm BS, Terdening CT, Benz DJ et al. Estrogen binding receptor mRNA, and biologic Response in osteoblast-like osteossarcoma cells. Science 2005; 24: 81-83.

14. Moreira C. Osteoporose. In: Rocha MO, et al. Terapêutica clínica. Rio de Janeiro: Guanabara Koogan; 1998. p. 588-604.

15. Mundy GR. The effects of cytockines and growth factores on osteoblast cells. Bone. 1995; 2 (7): 71-75.
16. Narayana MPS, Sengupta S, Shama S, Singh MM. Effect of ormeloxifene on ovariectomy-induced bone resorption, osteoclast differentiation and apoptosis and TGF beta-3 expression. J Steroid Biochem Mol Biol. 2006; 100 (4-5): 117-28.

17. Oliveira LAA. Avaliação do efeito dos risedronato sódico na consolidação das fraturas: Estudo experimental em ratos [tese]. São Paulo: Faculdade de Medicina, Universidade de São Paulo; 2003.

18. Otomo H, Sakai A, Ikeda S, Tanaka S, Ito M, Phipps RJ, Nakamura T. Regulation of mineral-to-matrix ratio of lumbar trabecular bone in ovariectomized rats treated with risedronate in combination with or without vitamin K2. J Bone Miner Metab. 2004; 22(5): 404-14.

19. Piai CR, Carvalho VAP, Werkman C, Anbinder AL, Rocha RF. Efeito do risedronato na reparação óssea de ratos machos e fêmeas com osteopenia. Cienc Odontol Bras. 2005 Jun/Set; 8 (3): 77-82.

20. Rang PH, Dale MM, Ritter JM, Moore PK. Farmacologia. 5.ed. Rio de Janeiro: Elsevier, 2003. 904p.

21. Reginster JY, Sarkar S, Zegels B, Henrotin Y, Bruyere O, Agnusdei D, et al. Reduction in PINP, a marker os bone metabolism, with raloxifene treatment and its relationship with vertebral fracture risk. Bone. 2004; 34(2) 344-51.

22. Rogers A, Clowes JA, Pereda CA, Eastell R. Different effects of raloxifene and estrogen on interleukin- $1 \uparrow^{2}$ and interleukin- 1 receptor antagonist production using in vitro and ex vivo studies. Bone. 2007; 40 (1): 105-10.

23. Sliwinski L, Folwarczna J, Janiec W, Grynkiewicz G, Kuzyk K. Differential effects of genistein, estradiol and raloxifene on rat osteoclasts in vitro. Pharmacol Rep. 2005; 57: 352-59.

24. Somjen D, Shen M, Stern N, Mirsky N. Diabetes modulates differentially creatine kinase-specific activity responsiveness to estradiol17beta and to raloxifene in rat organs. J Cell Biochem. 2006 Sep; 99 (1): 133-39.

25. Souza DM, Rosa LP, Ricardo LH, Moraes LC, Rocha RF. Avaliação óssea alveolar de rattus norvegicus por meio dos métodos radiográficos e morfométricos. Cienc Odontol Bras. 2005 Out/Dez; 8(4): 77-84.

26. Szejnfeld VL. Osteoporose: diagnóstico e tratamento. São Paulo: Savier; 2000. 393p.

27. Vieira S. Bioestatística: tópicos avançados. 2.ed. Rio de Janeiro: Elsevier; 2004.

Recebido em: 31/01/07

Aprovado em: 29/07/07

Valéria Abrantes Pinheiro Carvalho Avenida Adhemar de Barros, no 510 - apto 1202 Vila Adyanna - São José dos Campos - SP CEP - 12.245-011 Tel: (12) 3922.0402

E-mail - valeria@fosjc.unesp.br 